\title{
Modeling Bank of Kigali Stock Risks in Rwanda Stock Exchange Using Extreme Value Distribution
}

\author{
Katu Daniel Edem ${ }^{1 *}$, Marcel Ndengo ${ }^{2}$ \\ ${ }^{1}$ African Institute for Mathematical Sciences (AIMS), Kigali, Rwanda \\ ${ }^{2}$ Department of Natural Sciences, College of Arts and Sciences, William V.S. Tubman University, Harper, Liberia \\ Email: *danieledem1220@gmail.com, kdaniel@aims.ac.rw
}

How to cite this paper: Edem, K. D., \& Ndengo, M. (2021). Modeling Bank of Kigali Stock Risks in Rwanda Stock Exchange Using Extreme Value Distribution. Journal of Financial Risk Management, 10, 225-240. https://doi.org/10.4236/jfrm.2021.103013

Received: June 1, 2021

Accepted: July 31, 2021

Published: August 3, 2021

Copyright ( 2021 by author(s) and Scientific Research Publishing Inc. This work is licensed under the Creative Commons Attribution International License (CC BY 4.0).

http://creativecommons.org/licenses/by/4.0/

(c) (i) Open Access

\begin{abstract}
Extreme Value Theory has come forth as one of the most significant probability theories in applied sciences. Modeling extreme events has always been of interest in many disciplines such as hydrology, insurance, and finance. This study seeks to model the Bank of Kigali's (BK) stock risks in Rwanda stock exchange using Extreme Value Distribution. Two major approaches are used. To model Bank of Kigali stock risks, the Generalised Extreme Value Distribution (GEVD), precisely the Block Maxima is implemented. To examine its associated exceedances, the Generalised Pareto Distribution (GPD) is also implemented. Risk measures considered are the Value at Risk (VaR) and the Expected Shortfalls (ES). Findings reveal that the Frechet distribution fits reasonably well the distribution of the BK stock returns and GPD the exceedances. Also, the risk measures such as Value at Risk and Expected shortfall were computed with high level (99.5\%) quantiles to serve as a guide to investors to make a decision as to whether to invest in Bank of Kigali's stock or not. The findings show that GPD fits the tail of the data well.
\end{abstract}

\section{Keywords}

Extreme Value Theory, Generalised Extreme Value Distribution, Block Maxima, Generalised Pareto Distribution, Peak over Threshold, Value at Risk, Expected Shortfall

\section{Introduction}

Extreme Value Distribution has come forth as one of the most significant probability theories for social and applied sciences. Extreme Value Distribution gives the firm a strong foundation for building statistical models that tackle extreme 
events. EVD theory is widely used in many disciplines such as structural engineering, hydrology, traffic projections, insurance, and finance. EVT has the capability of determining the distribution of both maximum and minimum values. EVT is used to model the behaviour of maxima and minima of extreme tail loss of the distribution (Ramadhani et al., 2017). Taking into consideration, the probability of rare and extreme events, EVT provides a firm establishment needed for statistical modeling of extreme risk measures (Silva et al., 2011). The last decades have been exposed to instabilities in financial markets worldwide and for that matter, Rwanda. There comes the need to require tools to actually assess the probability of rare financial extremes. Different periods of financial markets have been affected by several crises such as the Stock Market Crash (1987), the Crisis (1997-1998) and Global Financial Crisis (2007-2008). The Global Financial Crisis in 2008, for example, led to an unforgettable mark of unemployment and debt. Then the question one will ask is what the right thing being done in finance? What was the cause for this? What went wrong? It is for these reasons that prompted looking for appropriate ways to deal with rare events that have a big effect on financial market (Chou \& Wang, 2014). In the heat of these financial disasters and crises, it has become clear to both risk managers and policymakers can be maintained or changed to risk managers and decision-makers. Extreme value theory has become one of the concerned studies in finance and insurance. (Embrechts et al., 2013) in his paper showed that extreme value theory methods fit the tails of heavy-tailed financial time series better than more conventional distributional approaches. He later recommended EVT to be the best approach in estimating the tail of loss distribution. In 1990s, due to the currency crisis and sub-primes in 2007, stock market crashes and credit default, a research study by (Gilli et al., 2006) showed the potential of EVT approach in finance and illustrated EVT using Block Maxima Method (BMM) and Peak Over Threshold (POT) in modelling VaR, ES and return level. His outcome depicted that POT was considered to be more efficient in modelling limited data and not depending on the requirement for large data set as BMM because it exploits better information in sampling (Murenzi et al., 2015). (de Dieu Ntawihebasenga et al., 2014) both returns and residuals have fat tail behaviour and concluded that GARCH-EVT approach can be worked very well. Another study on volatility analysis of exchange rate of emerging economies: a case of East Africa community showed that the existence of high rates of exchange volatility could be explained by the fact that these currencies are not pegged to any major international currency (Silva et al., 2011). Another illustration is a paper from (Tolikas \& Brown, 2006) where the authors used the Extreme Value Theory (EVT) to research the asymptotic distribution of the lower tail of daily returns in the Athens Stock Exchange (ASE) over the period 1986 to 2001. In this examination, they utilized GL distribution and discovered that it provides an adequate description of the ASE index daily returns minima. Its asymptotic convergence was found to be relatively stable, especially when large selection intervals were used. This is an 
imperative discovery since current EVT applications in finance focus exclusively on either the GEV or GPD distributions. These implications for investors could be important since the GL is fatter tailed than its GEV and GPD counterparts, which infers higher probabilities of extreme occurrences. (Ergen, 2010) discovered that the most successful methods in modeling fat tails and skewness are the EVT. (Gencay \& Selcuk, 2004) in their paper examined the relative performance for market risk models for the daily securities exchange returns of nine diverse emerging markets using EVT to generate tail risk estimates and came with the result that those tail risk estimates are more accurate at higher quantiles. Using the U.S stock market, (Longin, 2005) indicated how EVT can be valuable in adapting absolutely the characteristics of the distribution of asset returns, and finally help to select a better model by focusing on the tails of the distribution (Mwamba et al., 2017). (Nortey et al., 2015) in his paper wrote on how to empirically examine the application of the EVT process in the Ghana stock market by applying it to the Ghana Stock exchange all shares indices and turned out with the outcomes that, the daily returns of the Ghana stock exchange all-shares index data from a distribution are fat-tails and asymmetric in nature. Hence the Extreme Value Theory (EVT) model provided a better fit to the tails of the distribution of returns. However, due to the result of the observed volatility in the daily returns data, he used the conditional EVT approach for the study. (Seymour \& Polakow, 2015), additionally completed a comparative work by looking at comparing conditional and unconditional approaches in modelling the volatility of South Africa stock market and realized that the conditional approach provided a better result compared to unconditional approach. (Bi \& Giles, 2007) likewise inferred that the GPD performs extremely well in modelling both the positive and negative returns of the tail's distribution. (Daníelsson et al., 2000) on the caption, forecasting extreme financial risk: a basic examination of practical methods for the Japanese market utilizing EVT found that VaR forecasts are very accurate and stable after some time. This suggests that the use of EVT risk forecast for Japanese financial institutions and other users of Japanese market data are recommended. EVT approach is developed in the field of a probability of extreme events of a given distribution function. EVT has been accredited to be a wonderful tool that is widely used in measuring extremes and VaR estimation. EVT models heavy-tailed loss severity distribution (McNeil \& Saladin, 1997). There are two major approaches to measuring extreme values. The first one is the Block Maxima (BM) Method which happens to be the oldest group of a model. This approach considers a large observation collected from large samples of identically distributed observation. In many papers, it was observed that many take for granted that maxima follow very well on extreme value distribution. There is an important purpose of considering BM approach: The only available information may be BM for example yearly maxima with long historical records and the BM method may be preferable when the observations are not exactly independent and identically distributed (iid). For example, there may be 
a seasonal periodicity in case of yearly maxima. The second one is the Peak Over Threshold (POT) method. In POT model, the excess losses above a high threshold are modelled with GPD (McNeil \& Saladin, 1997). The POT method is preferred to be the best. This is because it has been proven to effectively and efficiently utilize more of the data and also produce useful findings (Nortey et al., 2015). These two approaches have some similarities and differences. The similarity is that POT and BM often have comparable performances for example for large sample sizes. The difference is POT is more efficient than BM in many circumstances. On average one considers exceedances larger than the number of blocks (Ferreira et al., 2015). Even though there are many published papers contributing to modeling bank's or financial risk using extreme value distribution, the majority of these papers focused on developed world. Many tend to focus on modeling individual investors in developed market such as USA, Western Europe, Asia, Japan, etc. Furthermore, there are few studies about financial modeling in developing countries like Rwanda. Studies, for example, (Mahina et al., 2017) focused on the Influence of Loss Aversion Bias on Investments at the Rwanda Stock-Exchange also, turned out with the findings that stock market tends to be more regretful about holding losing stocks too long than offering winning ones too early. This is because many stock market investors' failure depresses them (Mahina et al., 2017). However, this essay will be quite different as it will analyze the level of excellence and usage of Extreme Value Distribution for modeling Bank of Kigali stock risks in Rwanda stock exchange. Vision 2020 is the hallmark of Rwanda's development whereby it presents the key priorities and provides Rwandans a guiding tool for the future. The government of Rwanda together with its partners, donors, civil society organizations and private sector are now working assiduously to reaching the goal of vision 2020 (Kaberuka et al., 2000). Due to this, the government of Rwanda has encouraged participation and growth of capital in the stock market (Mauwa et al., 2017). In spite of these efforts, investment in Rwanda stock exchange is still low and the Rwanda stock exchange is not growing as much as expected. Few studies have been conducted in relation to RSE. (Kaberuka et al., 2000) wrote on the influence of loss aversion bias on investment at the RSE and came out with a result that loss aversion bias significantly affects investment in RSE. However, it appears that no studies have been done on modeling any of the stocks risk in RSE using EVT. Hence, this study will seek to fill this gap, which is to model BK stock risks with EVD.

\subsection{General Objectives}

The main objective of this essay is to model Bank of Kigali stock risks in Rwanda stock exchange using extreme value distribution.

\section{Specific Objectives}

The specific objectives in this work include:

1) Fitting Bank of Kigali daily losses with GEVD

2) Determining the exceedances by fitting the daily losses with the GPD 
3) Assessing the stock's value at risk (VaR)

4) De-clustering the exceedances

\subsection{Research Questions}

The research questions to this essay are:

1) Which particular case of the GEVD could fit reasonably well the BK daily stock losses?

2) Which size of exceedances are associated with the BK daily losses when GPD is used?

3) What is BK stocks VaR size?

4) To what extent can the de-cluster the exceedances be informative of the behaviour of VaR and ES?

\section{Theoretical Review}

\subsection{Extreme Value Distribution (EVD)}

Notations: $X$ is the random variable assumed to be independent and identically distributed (iid), $F(X)$ is a distribution function, $M_{n}$ is Maxima of $n$ observations with $P_{r}(X)$ being the probability function. $F^{n}$ denotes nth power of distribution function. $H(x)$ is non-degenerated distribution function.

$X_{F}$ signifies the right endpoint of $F, X_{1: n}$ is the sample size $n$ drawn from a population and $\mu$ is the threshold.

\subsubsection{Maxima and Minima Distribution}

Let $X_{1}, X_{2}, \cdots, X_{n}$ can be changed to be independent and identically distributed (iid) random variable which has a common distribution function $F(X)$ with some fixed $n$ by definition:

$$
M_{n}=\max \left(X_{1}, X_{2}, \cdots, X_{n}\right)
$$

is the maxima of $n$ observations and $M_{n}$ has distribution function denoted by $F_{n}$. In principle, if the distribution function for $X_{i}$ is assumed to be known, then the distribution of $M_{n}$ is derived as follows:

$$
F_{n}(x)=P_{r}\left(M_{n} \leq x\right)=P_{r}\left(X_{1} \leq x, X_{2} \leq x, \cdots, X_{n} \leq x\right)
$$

Since the observations are assumed are assumed to be independent.

$$
\begin{aligned}
P_{r}\left(M_{n} \leq x\right) & =P_{r}\left(X_{1} \leq x\right) \times P_{r}\left(X_{2} \leq x\right) \times \cdots \times P_{r}\left(X_{n} \leq x\right) \\
& =[F(x)]^{n} \\
& =F^{n}(x)
\end{aligned}
$$

The three-distribution family that belongs to Generalized Extreme Value Distribution are Weibull, Gumbel, and Frechet distribution. Weibull distribution appears practical when we observe data that deal with minima values. The CDF of Weibull random variable is given by

$$
F(x)=1-\exp \left[\left(-\frac{x-\mu}{\delta}\right)^{k}\right], x \geq \mu
$$


Gumbel distribution appears practical when we observe data that deal with maxima values. The CDF of Gumbel random variable is given by:

$$
F(x)=\exp \left[-\exp \left(\frac{x-\mu}{\delta}\right)\right],-\infty<x<\infty
$$

Frechet distribution appears practical when one observes data that deal with maxima values. The CDF of Frechet random variable is given by:

$$
F(x)=\exp \left[-\left(-\frac{x-\mu}{\delta}\right)^{k}\right], x>\mu
$$

All these distributions are particular cases of the maximal GEVD.

\subsubsection{Generalized Pareto Distribution}

The Generalized Pareto Distribution (GPD) was introduced by (Castillo, 2005). When one regards an unknown distribution function $F$ of a random variable $X$, the researcher will be interested in estimating the distribution function $F_{u}$ of a variable of $x$ above a certain threshold $\mu . F_{u}$ can be defined as the excess distribution function and it is given as:

$$
F_{U}(y)=P(X-\mu \leq y \mid X>\mu), 0 \leq y \leq X_{F}-\mu
$$

where $X$ is the random variable, $\mu$ is the given threshold, $y=x-\mu$ are the excesses. $X_{F}$ is the right endpoint of $F$. We can write $F_{U}$ as in (Altar et al., 2015).

$$
F_{U}(y)=\frac{F(\mu+y)-F(\mu)}{1-F(\mu)}=\frac{F(x)-F(\mu)}{1-F(\mu)}
$$

The GEVD alongside its specificities (Weibull, Frechet, and Gumbel distribution) have been introduced: its quantiles discussed and the method of estimating the parameter briefly spelled out. To deal with both exceedances and shortfall, the GPD has been introduced.

\section{Research Design and Methodology}

\subsection{Data Description}

In this work, the adjusted closing daily stock market prices for Bank of Kigali investment in the Rwanda Stock Exchange are used. The sample consists of 1226 daily closing prices of RSE starting from 3rd January 2012 to 16 November 2017. 2012-2017 closing prices because those were the data available as at the time the researcher was conducting the study. There are 229 missing data or $18.7 \%$ of the total data collected. This is not strange because according to (Oh, 2015) most financial stock market data, for various reasons, frequently contain missing values. The various reasons for missing data in stock market could be loss of information because of market close for holidays, daily stocks are not always observed. Missing prices are imputed via na.interpolation method, precisely the polynomial spline. Computation tool used is the R software version, 3.4.4. 


\subsection{Research Design}

Quantitative research was used to describe procedures that are followed to model Bank of Kigali stock risks using the GEV distribution. To identify the threshold, the GPD is used. The following steps are performed: 1) Observation of stock prices from RSE in terms their daily returns; 2) Cleaning of data and imputation of missing stock prices using appropriate smoothing method, precisely, na.interpolation (spline); 3) Data Processing using R software, version 3.4.4. With the help of R software, dataset is processed to: 1) Measure bank of Kigali stock risks using GEVD specifically using Block Maxima approach; 2) Determine the exceedances by fitting the daily loses using GPD precisely Peak Over Threshold; 3) Assess the measures of risk (VaR and Es). 4) De-cluster the exceedances occurring in the stock's returns. iv. Results from data processing shall be displayed in figures, tables and discussed in details in line with our specific objectives.

\subsection{Methodology}

In fitting the GEVD discussed to data as described. Two approaches are used, mainly the Block Maxima method and the Peak over Threshold. Block Maxima theory deals with the convergence of maxima, that is, the limits law for the maxima.

\subsubsection{Block Maxima}

To actually illustrate this, assume that $r_{t}, t=1, \cdots, n$, is a sequence of iid observations which have a distribution function $H(x)=P_{r}\left(r_{t} \leq x\right)$ and let the sample maximum be denoted by $M_{n}=\max \left\{r_{1}, \cdots, r_{n}\right\}$ where $n \geq 2$ and $R$ denotes the real line.

GEVD represented by $H(x)$ describes the limiting distribution of suitably normalised maxima.

The random variable $X$ may be replaced by $\frac{x-\mu}{\delta}$ to get a standard GEV with distribution function where $\mu, \delta$, and $k$ are the location, scale, and shape parameters respectively. The function $\mathrm{H}$ may belong to one of the three standard extreme value distributions.

Frechet: $\varphi(x)= \begin{cases}-x, & x \leq 0 \\ \exp (-x)^{-\alpha}, & x \geq 0\end{cases}$

Weibull: $\psi(x)= \begin{cases}\exp -\left\{(-x)^{\alpha}\right\}, & \alpha \leq 0 \\ 1, & x \geq 0\end{cases}$

Gumbel: $\varphi(x)=\exp \left\{(\exp )^{-x}\right\}, \quad x \in R$

\subsubsection{Peak over Threshold}

Considering Peak over Threshold, the focus is on set of realized values exceeding a certain threshold. One technique of removing extremes from a sample of observations $r_{t}, t=1,2, \cdots, n$ with distribution function $F(x)=P_{r}\left(r_{t} \leq x\right)$ is to 
take exceedances over a deterministic high threshold $\mu$. Exceedances over threshold occur when $r_{t}>\mu$ for any $t$ in $t=1, \cdots, n$. An excess over $\mu$ is defined by $x=r_{i}-u$. Given a threshold $\mu$, the probability distribution of excess values of $r_{t}$ over threshold is defined as $F_{u}(x)=P_{r}\{r-\mu \leq x \mid r>\mu\}$. This represent the probability that the value of $r$ exceeds the threshold $\mu$ by at most an amount $X$ given that $r$ exceeds the threshold $\mu$.

Measuring risks use VaR and ES. The mathematical tool to deal with risk measures are the Value at Risk (VaR), Expected Shortfall (ES). VaR is a tool for risks assessment. Its utilization in Banks reflects their fear of a liquidity emergency, where a low-probability catastrophic occurrence is made. Assume an arbitrary variable $X$, with continuous distribution function $F$, models return of an asset over a certain time horizon. The $\mathrm{VaR}_{p}$ is the $p^{\text {th }}$ quantile of the distribution $F$ defined as $\operatorname{VaR}_{p}=F^{-1}(1-P)$, where $F^{-1}$ is the $P^{t h}$ quantile function.

The ES is defined as the expected size of a return that exceeds $\mathrm{VaR}_{p}$.

$$
\begin{gathered}
\mathrm{ES}_{P}=E\left(X \mid X>\mathrm{VaR}_{p}\right) \\
\mathrm{ES}_{\alpha}=\frac{1}{1-\alpha} \int_{\alpha}^{1} q_{x}(F) \mathrm{d} x=\frac{\operatorname{var}_{\alpha}}{1-\varepsilon}+\frac{\delta-\varepsilon_{\mu}}{1-\varepsilon} \\
\operatorname{VAR}_{\alpha}=q_{\alpha}(F)=\mu+\frac{\delta}{\varepsilon}\left[\left(\frac{1-\alpha}{1-F(\mu)}\right)^{-\varepsilon}-1\right]
\end{gathered}
$$

$F(\mu)$ denote the number of non-exceedances relative to the sample size (McNeil \& Saladin, 1997).

\subsubsection{Declustering}

Declustering systems are strategies that look to distinguish occasions in a data record and filter out extreme values from them. The maxima excess in each cluster is identified, and these cluster maxima can be assumed to be independent. The GPD would then be able to be fitted to the cluster maxima. Declustering happens as a method for data preprocessing. The purpose of this data preprocessing is to ensure the validity of the GPD assumptions (Bommier, 2014).

\section{Results and Discussion}

This chapter introduces results and discussion of the various methods used to meet the specific objectives above. The main focus is on using Block Maxima method, Peak over Threshold, Value at Risk, and Expected Shortfall to model the stocks returns.

\subsection{Data Description and Imputing of Missing Data}

When a dataset is given, it is expedient to assess its overall quality. This exercise has been done thoroughly. Data collected from RSE source was checked. However, the dataset was quite inconsistent.

Raw Data: The raw data of Bank of Kigali stock prices over a year is displayed 
in Figure 1.

A closer look at Figure 1, one notices existence of some gaps, or discontinuity of the graph. This signifies missing values in the dataset. Prior to any analysis, imputation of missing values is performed.

In Figure 2, the imputation method (nainterpolation) was used for obtaining the missing data using the splines. After the imputation method, it is observed that all the gaps are filled and modeling of the stock returns can now proceed with the complete dataset as can be seen in Figure 2.

\subsection{Block Maxima}

To measure stock losses, the GEVD is used in the Block Maxima configuration and numerical results are displayed in Table 1 , for parameter estimates for daily losses in Figure 3 and finally the diagnostic plots in Figure 4.

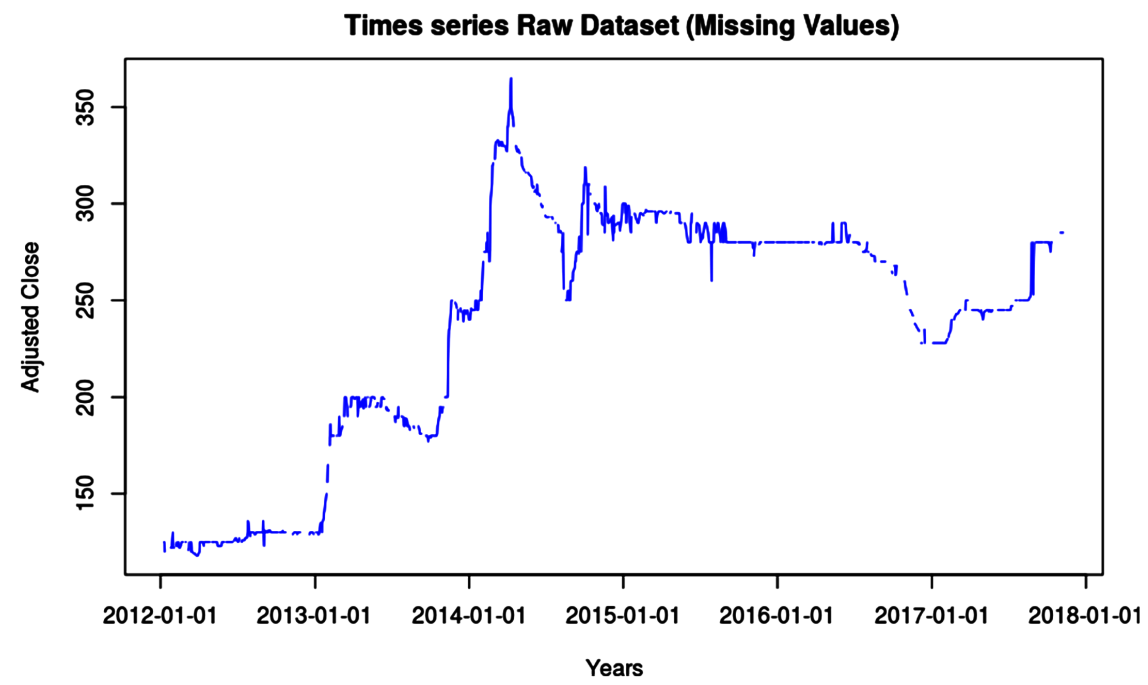

Figure 1. Time series raw dataset (Missing values).

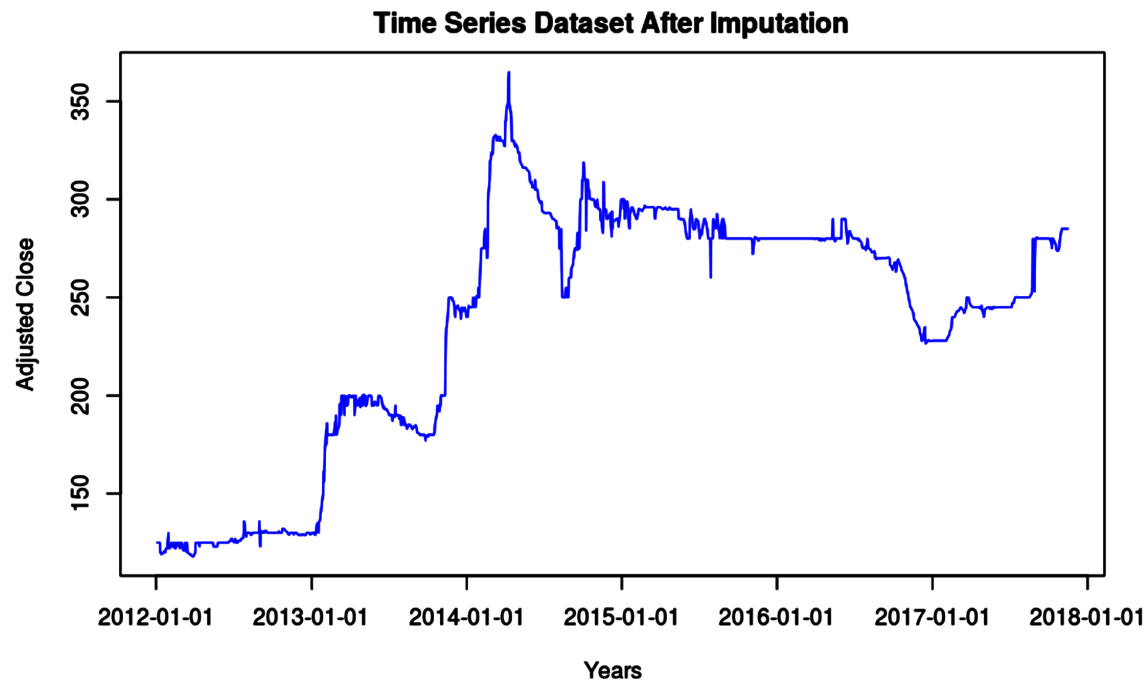

Figure 2. Time series data set after imputation. 


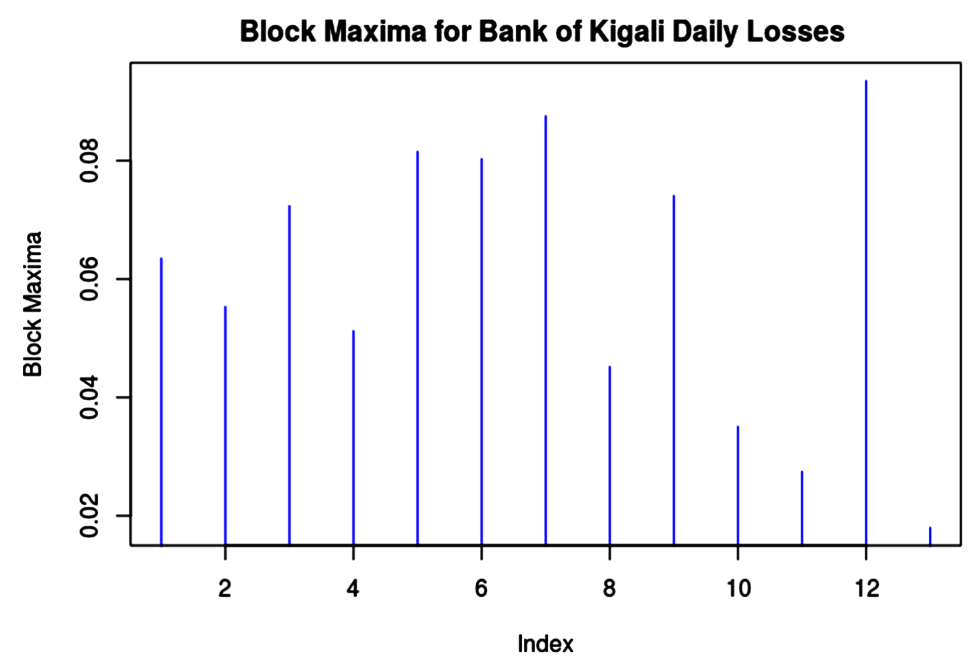

Figure 3. Block maxima depicting BK daily loses.
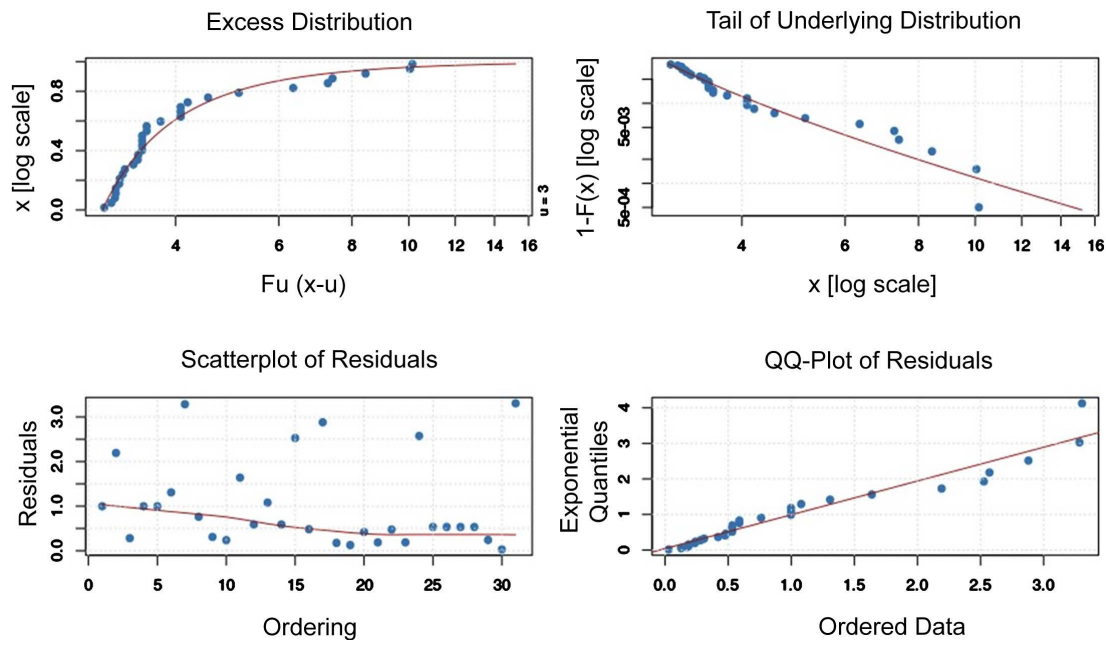

Figure 4. Diagnostic plot for GPD model.

Table 1. Generalised extreme value estimates for bank of Kigali.

\begin{tabular}{cccc}
\hline GEV & $K$ & $\Delta$ & $\mu$ \\
\hline Estimates & -0.526 & 0.73 & 2.41 \\
Standard error & 0.1916 & 0.170 & 0.222 \\
\hline
\end{tabular}

To actually demonstrate fitting the GEV distribution to block maxima data, we used Bank of Kigali data set. As block maxima deal with dividing the observations into periods of non-overlapping equal sizes and paying attention to the maximum in each period, the block size was divided into 100 observations. As a result, a total sample size of 13 observations was obtained, each containing the daily returns of a subsequent month, as it can be seen in Figure 3.

In Table 1, the Maximum likelihood (ML) estimates of parameter are presented. It can be seen that all the coefficients are significantly different from zero. Shape parameter is a helpful tool for checking if a given distribution is ap- 
propriate or not. Considering the shape parameter $\mathrm{k}$ from the current analysis, since $k<0$, it follows that the GEV is of Frechet type. It can also be said that there is an existence of heavy-tail since the higher the absolute value of the shape parameter the heavier and weightier the tail and the slower the speed at which the tail approaches its limit. Computing the skewness and kurtosis we had 0.497 and -1.144 respectively. This depicts that the distribution of the return data is fat-tailed. Also, since the shape operator, $k$ is less than zero, $F(x)$ belongs to the Frechet minimal domain of attraction.

Figure 5 depicts diagnostic plot for fitting GEV to the block maxima data of sample size of 13. It can be seen that the top two plots do not deviate much from the straight line. Empirical observations (marked with a circle) are close to the diagonal, representing a perfect fit in both the PP and QQ plot. For the density plot, it can be observed that the curve does not really match up with the histogram very well. However, it can be seen that it is left-skewed (negative skewness). The return level presents a graph of the empirical estimates of the return level plotted against the estimated return levels from the fitted model. It can also be observed from the plot that all the points lie approximately on the line.

The shape and scale parameter are presented in Table 2 below. From Table 2, it can be seen that the shape parameter $k$, is greater than zero and it is significantly different from the one which indicates fat tail or heavy tail distribution. However, the parameters changes depending on the value of the threshold.

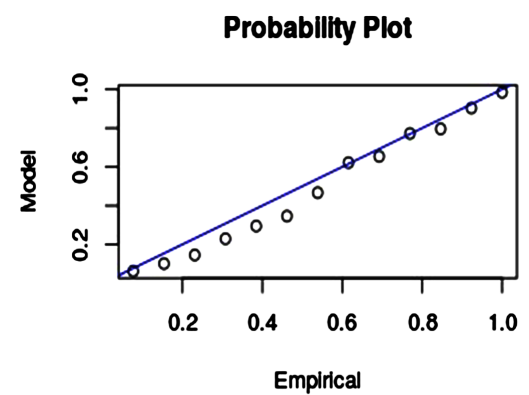

Return Level Plot

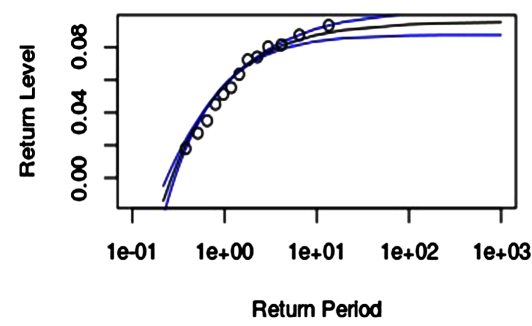

Quantlle Plot

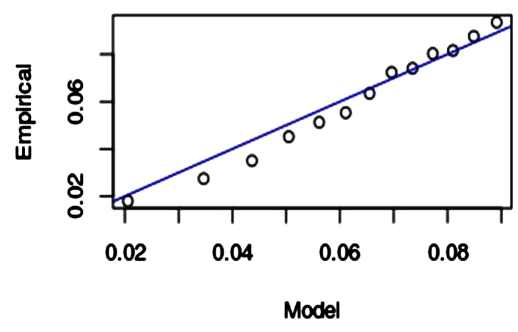

Density Plot

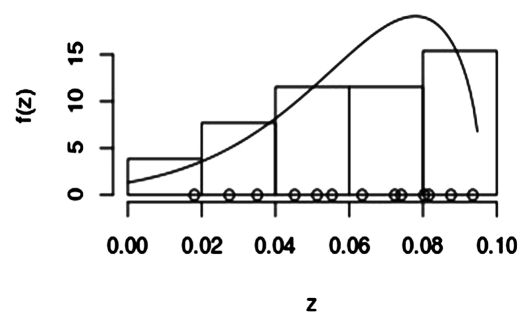

Figure 5. Diagnostic plot for fitting GEV.

Table 2. Fitted GPD of BANK of Kigali stock prices.

\begin{tabular}{ccc}
\hline GPD & $k$ & $\delta$ \\
\hline Estimates & 0.507 & 0.833 \\
\hline
\end{tabular}


The $\mathrm{VaR}$ and ES risk measures are computed for 95\%, 97\%, 99\%, and $99.5 \%$ levels. The outcomes are displayed in Table 3. VaR and ES are tools for risk assessment and also help in assessing the next business day.

From Table 3, it can be seen that at 95\% confidence level, the next business day is likely not to exceed $2.65 \%$ Rwanda Francs (Rwf) and that the average gain in situations where gains exceed $2.65 \%$ is $3.98 \%$ Rwf. With $97 \%$ confidence level, the next business day is likely to a loss $3.03 \%$ Rwf and the corresponding expected gain in a situation where gain exceeds $3.03 \%$ is $4.76 \%$. Similarly, at $99.5 \%$ confidence level, the next business day is likely to a loss $5.51 \%$ Rwf and the corresponding expected gain in a situation where gains exceed $5.51 \%$ is $9.8 \%$.

From Figure 4, the upper panels demonstrate the fitted excess distribution and a tail plot. Both show a good fit of the GPD to the exceedances. It is a good fit because it does not deviate too much from the straight line except for one data point. The lower panel displays the residuals with a fitted ordinary least square (OLS) line on the left and Q-Q plot also does not deviate from the true line. With these, it is clear that POT is a good measure as it produces useful findings.

Figure 6 shows how Bank of Kigali data set are clustered. POT method requires the exceedances to be mutually independent. In order to deal with the independent variables and able to apply the POT method, a commonly used technique is declustering. From the graph, it can be seen that 2013 and 2017 experiences higher exceedances. This is because those periods exceeded the threshold causing a higher variance at the extreme payouts in the data which are larger than the average point.

It is necessary for dataset to perform declustering in order to estimate the parameters $k$ and $\delta$. The estimates are shown in Table 4 . Table 4 shows declustering into weekly, bi-weekly, and monthly accompanied by the estimates $k, \delta$, and the exceedances. It can be observed that the shape parameter $k$, which defines the behaviour of the tail of the GPD decreases as the number of runs increases. Also, the scale parameter which measures the variability of the distribution shows an increasing nature as the number of runs increases. One must note that, as means of data preprocessing, the exceedances ought to be de-clustered. However, only the extreme is recovered within a cluster of exceedances as the representation of maximum loss which from Table 4 is 47 . The point of this data preprocessing technique is to ensure the validity of the GPD assumptions. One must note that the outcomes are delicate to the decision of the number of runs. By way of fitting the GPD to these series is the outcome as can be seen in Table 4.

Table 3. Risk measures for bank of Kigali stock.

\begin{tabular}{ccccc}
\hline Confidence level & $95 \%$ & $97 \%$ & $99 \%$ & $99.5 \%$ \\
\hline VaR & 2.65 & 3.03 & 4.28 & 5.510 \\
ES & 3.98 & 4.76 & 7.29 & 9.786 \\
\hline
\end{tabular}




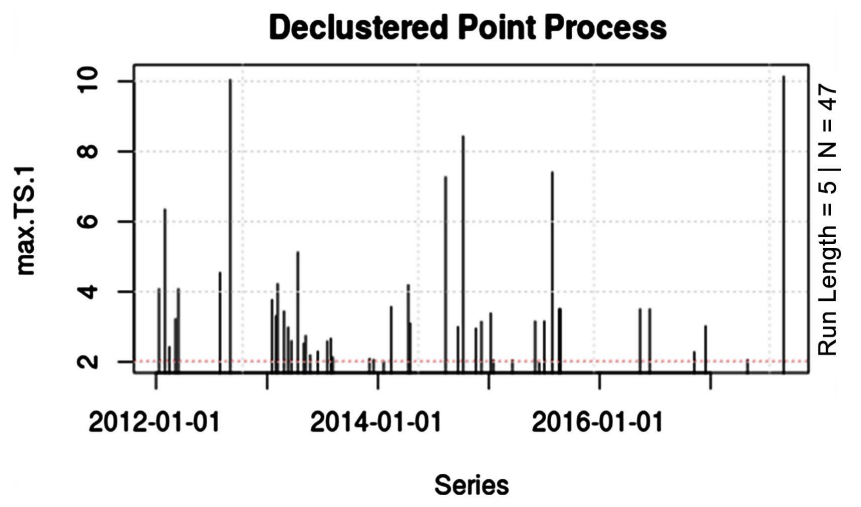

Figure 6. Plot for clustering of BK stock exceedances.

Table 4. Results for Declustered GPD models.

\begin{tabular}{rccc}
\hline Decluster & $K$ & $\delta$ & Exceedances \\
\hline Weekly & 0.163 & 1.453 & 47 \\
Bi-weekly & 0.13 & 1.71 & 38 \\
Monthly & -0.06 & 2.64 & 25 \\
\hline
\end{tabular}

\section{Conclusions and Recommendation}

\subsection{Conclusion}

This study showed how Extreme Value Theory can be a useful tool to model Bank of Kigali's stock risks. Also, it was revealed how EVT can be used to model tail-related measures such as Value at Risk, expected shortfall and applying it to Bank of Kigali stock prices. The study exhibited heavy-tail behaviour in returns of the Bank's data and provided a better fit to the tails of the distribution of results. From the study, it was observed that the GEV estimates provided all the coefficients to be significantly greater than zero. The shape parameter for instance, which is a useful tool for checking whether a given distribution is appropriate or not gave values less than zero, signifying GEV is of Frechet type. This demonstrates the existence of heavy-tail. Hence, EVT model provided a good fit to the tails of the distribution of returns. The domain of attraction also was seen to belong to the Frechet minimal domain of attraction. The study further observed the diagnostic plot where it was realized that the QQ plot does not deviate significantly from the straight line which signifies a good fit. The study employed POT model and came with the findings that POT provides better estimation to the extreme events as it dwells much on estimating the tail-end using Extreme value beyond a certain threshold, unlike BM which utilizes only the maximum losses in large blocks. The study also used the VaR and Es in determining how good or bad the market value of RSE is at a given level of confidence. The $\mathrm{VaR}$ and ES risk measures were computed for $95 \%, 97 \%, 99 \%$, and $99.5 \%$. It was observed that the VaR estimates were more accurate at higher quantiles. For instance, with $99.5 \%$ confidence level, the next business day is likely not to exceed $5.51 \%$ and the corresponding gain in a situation where gain exceed $5.51 \%$ is 
9.8\%. Considering the above results imply that for an investment of $\$ 1$ million in the market, the expected gains will not exceed $\$ 55,100$, with $99.5 \%$ confidence level. This serves as a guide to investors as they will invest in a stock when faced with sure gain. Both BM and POT are good approaches to the measure of Bank risks, however, POT is preferred in this study as it utilizes the information in the data obtained. This supports why EVT is used for extreme events. Some of the deficiencies of this paper are the availability of the data set without missing values as at the time the researcher was conducting this research. Also, one could combine two methods such as EVT and Copula approach or even the Garch Model. However, further work can be developed on this to look into using these methods.

\subsection{Recommendation}

The study recommends Rwandan investors, especially risk-averse ones, who would prefer not to take up risky investments in light of the propensity of losing their investments to go on and invest. This comes forth from the result of risk measures (VaR and ES) as obtained in the study. This is because VaR is used to quantify the risk of a portfolio over a specific time frame. VaR is a very useful tool for investment as it provides a means of assessing how risk exposure managers are taking on to achieve their portfolio return. One could have used the non-parametric estimating methods like the kernels, splines, since these estimating methods can estimate stocks without bias and help to obtain better forecasts. Studies also can be conducted on this distribution using the Kernel distribution instead of EVT. This work also can serve as a guide to insurance companies and financial economists in modelling risks associated with them.

\section{Acknowledgements}

The authors would like to thank the African Institute for Mathematical Sciences (AIMS) for their immense support and my supervisor Dr. Marcel Ndengo for making this work become possibility.

\section{Conflicts of Interest}

The authors declare no conflicts of interest regarding the publication of this paper.

\section{References}

Altar, M., Ifrim, A., Altar-Samuel, A. N., Chang, T. Y., Hao, F. A. N. G., Yen-Hsien, L. E. E., Yuchen, W., Dumitrescu, B. A. et al. (2015). Analysis and Surveys. Romanian Journal of Economic Forecasting, 18.

Bi, G., \& Giles, D. E. (2007). An Application of Extreme Value Analysis to Us Movie Box Office Returns. In Proceedings of the 2007: International Congress on Modelling and Simulation: Land, Water and Environmental Management: Integrated Systems for Sustainability (pp. 2652-2658). Citeseer.

Bommier, E. (2014). Peaks-over-Threshold Modelling of Environmental Data. 
Castillo, E., Hadi, A. S., Balakrishnan, N., \& Sarabia, J.-M. (2005). Extreme Value and Related Models with Applications in Engineering and Science (Vol. 25, 362 p). Wiley.

Chou, H.-C., \& Wang, D. K. (2014). Estimation of Tail-Related Value-at-Risk Measures: Range Based Extreme Value Approach. Quantitative Finance, 14, 293-304.

https://doi.org/10.1080/14697688.2013.819113

Daníelsson, J., Morimoto, Y. et al. (2000). Forecasting Extreme Financial Risk: A Critical Analysis of Practical Methods for the Japanese Market. Institute for Monetary and Economic Studies, Bank of Japan.

De Dieu Ntawihebasenga, J., Mwita, P., \& Mung'atu, J. (2014). Modelling the Volatility of Exchange Rates in Rwandese Markets. European Journal of Statistics and Probability, 2, 23-33.

De Sousa e Silva, J. et al. (2011). How to Deal with Extreme Observations in Empirical Finance: An Application to Capital Markets. Ph.D. Thesis, ISCTE Business School.

Embrechts, P., Klüppelberg, C., \& Mikosch, T. (2013). Modelling Extremal Events: for Insurance and Finance (Vol. 33). Springer Science \& Business Media.

Ergen, I. (2010). Var Prediction for Emerging Stock Markets: Garch Filtered Skewed $t$ Distribution and GARCH Filtered EVT Method. Working Paper.

Ferreira, A., De Haan, L. et al. (2015). On the Block Maxima Method in Extreme Value theory: PWM Estimators. The Annals of Statistics, 43, 276-298.

https://doi.org/10.1214/14-AOS1280

Gencay, R., \& Selcuk, F. (2004). Extreme Value Theory and Value-at-Risk: Relative Performance in Emerging Markets. International Journal of Forecasting, 20, 287-303. https://doi.org/10.1016/j.ijforecast.2003.09.005

Gilli, M. et al. (2006). An Application of Extreme Value Theory for Measuring Financial Risk. Computational Economics, 27, 207-228. https://doi.org/10.1007/s10614-006-9025-7

Kaberuka, D. et al. (2000). Rwanda Vision 2020. Republic of Rwanda Ministry of Finance and Economic Planning.

Longin, F. (2005). The Choice of the Distribution of Asset Returns: How Extreme Value Theory Can Help? Journal of Banking \& Finance, 29, 1017-1035. https://doi.org/10.1016/j.jbankfin.2004.08.011

Mahina, J. N., Muturi, W. M., \& Memba, F. S. (2017). Influence of Loss Aversion Bias on Investments at the Rwanda Stock Exchange. International Journal of Accounting, Finance and Risk Management, 2, 131-137.

Mauwa, J. et al (2017). Determinants of Financial Performance of Firms Listed on the Rwanda Stock Exchange. Doctoral Dissertation, COHRED, Jomo Kenyatta University of Agriculture and Technology.

McNeil, A. J., \& Saladin, T. (1997). The Peaks over Thresholds Method for Estimating High Quantiles of Loss Distributions. In Proceedings of 28th International ASTIN Colloquium (pp. 23-43).

Murenzi, R., Thomas, K., \& Mung'atu, J. K. (2015) Modeling Exchange Market Volatility Risk in Rwanda Using Garch-EVT Approach. International Journal of Thesis Projects and Dissertations, 3, 67-80.

Mwamba, J. W. M., Hammoudeh, S., \& Gupta, R. (2017). Financial Tail Risks in Conventional and Islamic Stock Markets: A Comparative Analysis. Pacific-Basin Finance Journal, 42, 60-82. https://doi.org/10.1016/j.pacfin.2016.01.003

Nortey, E. N., Asare, K., \& Mettle, F. O. (2015). Extreme Value Modelling of Ghana Stock Exchange Index. SpringerPlus, 4, Article No. 696. 
https://doi.org/10.1186/s40064-015-1306-y

Oh, S. (2015). Multiple Imputation on Missing Values in Time Series Data. Ph.D. Thesis, Duke University.

Ramadhani, F., Nurrohmah, S., \& Novita, M. (2017). Extreme Value Theory (EVT) Application on Estimating the Distribution of Maxima. In AIP Conference Proceedings (Vol. 1862, Article ID: 030156). AIP Publishing LLC. https://doi.org/10.1063/1.4991260

Seymour, A. J., \& Polakow, D. A. (2003). A Coupling of Extreme-Value Theory and Volatility Updating with Value-at-Risk Estimation in Emerging Markets: A South African Test. Multinational Finance Journal, 7, 3-23.

Tolikas, K., \& Brown, R. A. (2006). The Distribution of the Extreme Daily Share Returns in the Athens Stock Exchange. European Journal of Finance, 12, 1-22.

https://doi.org/10.1080/1351847042000304107 